\title{
Results from the ESO Distant Cluster Survey
}

\author{
Douglas Clowe $^{1}$, Peter Schneider ${ }^{2}$ and the EDisCS Consortium \\ ${ }^{1}$ Steward Observatory, 633 N Cherry Ave, Tucson, AZ 85712, USA email: clowe@as.arizona.edu \\ ${ }^{2}$ Institut für Astrophysik and Extraterrestrische Forschung, Universität Bonn, Auf dem Hügel \\ 71, D-53121 Bonn, Germany
}

\begin{abstract}
We present results from weak lensing analyses of 20 high-redshift clusters in the ESO Distant Cluster Survey (EDisCS) from both deep optical VLT and HST/ACS imaging. We find that the EDisCS sample is composed primarily of clusters that are less massive than those in current X-ray selected samples at similar redshifts, but that all of the fields are likely to contain massive clusters rather than superpositions low mass groups. We find good agreement between the weak lensing measurements in the VLT and ACS images. Finally we determine the noise level in the shear measurements for the VLT fields as a function of exposure time and seeing and demonstrate that future ground-based surveys which plan to perform deep optical imaging for use in weak lensing measurements must achieve point-spread functions smaller than a median of 0 .' 6 FWHM.
\end{abstract}

\section{Introduction}

The ESO Distant Cluster Survey (EDisCS) contains a sample of 20 high-redshift $(0.4<$ $z<0.9$ ) galaxy clusters, drawn from the optically selected Las Campanas Distant Cluster Survey (Gonzalez et al. 2001). Because this sample contains 20 clusters drawn from a 130 square degree survey, over which area X-ray surveys to the depth of EMSS and the ROSAT NEP would find $\sim 4$ clusters, one expects EDisCS to be made primarily of clusters at lower masses than those of contemporary X-ray surveys at these same redshifts.

The clusters have been observed with deep VLT/FORS2 optical imaging, NTT/SOFI infrared imaging, wide-field optical imaging with the WFI, and VLT/FORS2 multi-object spectroscopy. In addition, 10 of these clusters have been observed with HST/ACS mosaic imaging. In this paper we report on weak lensing analyses of these clusters from the optical VLT and ACS images.

\section{Data Reduction}

The EDisCS sample is divided into two section based on the expected redshift of the cluster from the LCDCS data. The moderate redshift sample $(z \sim 0.5)$ were observed with FORS2 in $I, V$, and $B$ passbands with 45 minutes of integration time per passband. The high redshift sample $(z>0.6)$ were observed with FORS2 in $I, R$, and $B$ passband with 120 minutes of integration time per passband. The final coadded images have PSF sizes which range from 0 ". 48 to 0 "' 85 . An ultra-deep field for each cluster where all three passbands were coadded was also created for use as the detection image for the peakfinder. The final image size was $\sim 7$ ! 3 , with $\sim 6$ ! 8 having the full exposure time.

Objects were detected in the ultra-deep field using SExtractor (Bertin \& Arnouts 1996) and had their photometry measured using the two-image mode. Moments of the surface brightness of the objects was measured using IMCAT, and the resulting ellipticities were 
corrected for PSF smearing using the KSB method (Kaiser, Squires, \& Broadhurst 1995). Shear measurements for each object were obtained in each passband separately, then combined into a final measurement using a weighted mean, with the square of the total significance of the object in the passband as the weight.

Ten of the clusters have also been observed with ACS, 9 from the high redshift sample and one from the moderate redshift sample. Each cluster was observed over 8 orbits in the 814 filter, with four orbits taken as a $2 \times 2$ mosaic covering the area of the VLT images and the remaining four orbits centered on the cluster core. Total exposure time was $\sim 10.2 \mathrm{ks}$ in the cluster core and $\sim 2.04 \mathrm{ks}$ in the outer regions. The images were reduced on the fly with CALACS v4.3, and we used Multidrizzle to remove cosmic rays, undistort the images, and drizzle them into a single mosaic image. Cross-correlation of the undistorted images was used to provide Multidrizzle with precise image offsets.

Objects were detected using SExtractor, had colors measured on the VLT images using a matched aperture, and had moments of the surface brightness measured using IMCAT. The resulting ellipticities were corrected for PSF smearing using the KSB method, but because of the mosaic nature of the images causes rapid changes in PSF shape we could not model the PSF directly from the combined image. Instead we built a model for the PSF with variations both spatially across the image and temporally with the observation date. We then traced where each object in the final image was in each input image, and built a composite PSF model, which was used to calculate the KSB correction factors.

\section{Cluster Mass Measurements}

Because the relatively small sizes of the field and the mass sheet degeneracy, we cannot measure more than a lower limit on the projected mass of the clusters in a non-parametric method. Instead, we have measured the cluster masses by fitting parameterized mass profiles to the azimuthally averaged shear profiles, using the brightest cluster galaxy of the cluster as the assumed center of mass. We fit a singular isothermal sphere, NFW, and a cored-King model to the clusters, but find that because of the small radial range over which we can fit, $\sim 100 \mathrm{kpc}<r<\sim 700 \mathrm{kpc}$, the two parameters in the NFW and King models were highly degenerate. As a result, only the SIS profiles provide a useful measure of the mass. N-body simulations have shown that over this range the integrated SIS profile does provide a good description of the integrated cluster mass profile to within the error in the shear measurements for these clusters.

The best-fit SIS profiles for each cluster, using the BCG as the assumed center of mass, and the significance of the fit, as measured by the $\delta \chi^{2}$ between the best fit and a $0 \mathrm{~km} / \mathrm{s}$ fit, are given in Table 1 . The mean redshift of the background galaxies assumed in the fits is also given in Table 1 and was calculated by applying the same magnitude and color cuts to the ground-based VLT photometry of the HDF-S photometric redshift catalogs from Fontana et al. (1999). The errors in the velocity dispersion are only the random errors from the shear measurement and do not include any uncertainty from the assumption of the mean redshift of the background galaxies. We also give in Table 1 the mass-to-light ratio of the clusters in rest-frame $I$.

We have also measured SIS fits to the 9 clusters with ACS mosaics, the results of which are in Table 1. The mean redshift of the background galaxies for these images was also estimated from the Fontana et al. (1999) HDF-S photometric redshift catalogs. The measured velocity dispersions from the two data sets are in good statistical agreement.

We show in Figure 1 the velocity dispersions for both sets of images plotted as a function of cluster redshift, along with weak lensing velocity dispersions of high-redshift X-ray selected clusters from Clowe et al. (2000) and lower redshift X-ray selected clusters from 
Table 1. Summary of weak lensing results. The columns show the cluster name, spectroscopic redshift, number density of background galaxies in the VLT images, mean lensing redshift of the background galaxies, best-fit SIS velocity dispersion with $1 \sigma$ errors, significance of the SIS fit, and the cluster mass-to-light ratio in $I$. In addition, clusters which have been observed with HST/ACS have the best-fit SIS velocity dispersion with $1 \sigma$ errors and the significance of the fit listed.

\begin{tabular}{lcccccccc}
$\begin{array}{l}\text { cluster } \\
\text { CL1018.8-1211 }\end{array}$ & 0.472 & 22.4 & 0.99 & $566_{-113}^{+90}$ & 2.7 & $215_{-77}^{+74}$ & & \\
CL1059.2-1253 & 0.457 & 21.4 & 0.97 & $826_{-119}^{+99}$ & 3.5 & $360_{-96}^{+92}$ & & \\
CL1119.3-1129 & 0.550 & 30.9 & 1.03 & $262_{-262}^{+188}$ & 0.5 & $147_{-147}^{+155}$ & & \\
CL1202.7-1224 & 0.424 & 25.6 & 0.96 & $504_{-103}^{+137}$ & 1.6 & $146_{-146}^{+280}$ & & \\
CL1232.5-1250 & 0.542 & 35.4 & 1.03 & $953_{-59}^{+54}$ & 7.6 & $203_{-24}^{+24}$ & $988_{-61}^{+57}$ & 8.4 \\
CL1238.5-1144 & 0.465 & 34.2 & 0.98 & $441_{-177}^{+120}$ & 1.6 & $180_{-114}^{+111}$ & & \\
CL1301.7-1139 & 0.485 & 36.4 & 0.99 & $634_{-96}^{+80}$ & 3.4 & $180_{-50}^{+48}$ & & \\
CL1353.0-1137 & 0.577 & 33.8 & 1.05 & $545_{-180}^{+131}$ & 1.8 & $124_{-68}^{+66}$ & & \\
CL1411.1-1148 & 0.52 & 34.4 & 1.01 & $566_{-158}^{+120}$ & 2.1 & $173_{-83}^{+81}$ & & \\
CL1420.3-1236 & 0.497 & 24.7 & 1.00 & $583_{-119}^{+97}$ & 2.7 & $283_{-104}^{+102}$ & & \\
CL1037.9-1243 & 0.580 & 54.2 & 1.11 & $570_{-102}^{+83}$ & 3.0 & $183_{-60}^{+57}$ & $511_{-134}^{+105}$ & 2.2 \\
CL1040.7-1155 & 0.704 & 44.4 & 1.19 & $266_{-266}^{+197}$ & 0.5 & $30_{-30}^{+61}$ & $484_{-173}^{+126}$ & 1.7 \\
CL1054.4-1146 & 0.697 & 38.4 & 1.18 & $883_{-119}^{+97}$ & 3.8 & $132_{-33}^{+31}$ & $756_{-96}^{+85}$ & 4.2 \\
CL1054.7-1245 & 0.750 & 35.8 & 1.22 & $969_{-102}^{+88}$ & 4.6 & $241_{-48}^{+45}$ & $1045_{-74}^{69}$ & 7.3 \\
CL1103.7-1245 & 0.660 & 32.5 & 1.16 & $710_{-163}^{+125}$ & 2.4 & $871_{-351}^{+334}$ & $430_{-154}^{+112}$ & 1.7 \\
CL1122.9-1136 & 0.807 & 34.9 & 1.26 & $435_{-435}^{+235}$ & 0.7 & $125_{-125}^{+172}$ & & \\
CL1138.2-1133 & 0.480 & 43.1 & 1.13 & $583_{-89}^{+74}$ & 3.4 & $229_{-64}^{+62}$ & $690_{-76}^{+68}$ & 4.8 \\
CL1216.8-1201 & 0.794 & 37.6 & 1.25 & $1173_{-85}^{+75}$ & 6.3 & $169_{-24}^{+22}$ & $956_{-86}^{+79}$ & 5.8 \\
CL1227.9-1138 & 0.634 & 31.1 & 1.14 & $265_{-265}^{+235}$ & 0.4 & $48_{-48}^{+123}$ & & \\
CL1354.2-1230 & 0.757 & 37.1 & 1.23 & $748_{-100}^{+85}$ & 3.9 & $297_{-75}^{+70}$ & $660_{-105}^{+91}$ & 3.4 \\
\hline
\end{tabular}

Dahle et al. (2002) and Cypriano et al. (2004). The EDisCS clusters are, as expected, of lower mass than X-ray selected clusters at similar redshift. From a comparison of the sky area covered by the EMSS and ROSAT NEP surveys, from which the high-redshift $\mathrm{X}$-ray selected sample was taken, to that of the LCDCS, from which the EDisCS sample was taken, we would expect on the order of 4 clusters in the EDisCS samples to have comparable masses to the X-ray sample. As can be seen in Figure 1, the EDisCS sample has 3-4 clusters with velocity dispersions similar to the high-redshift X-ray sample, in agreement with expectation.

Also shown in Figure 1 is a model for how the SIS velocity dispersions measured from weak lensing will evolve for a cluster with redshift. This model is based on the virial mass of the cluster growing at a $\propto \exp (-1.4 \times z)$ rate, which is in agreement with the growth rates of N-body clusters (Wechsler et al. 2002, Mo \& White 2002). We convert this virial mass into a measure of $r_{200}$ and $c$ for a cluster based on the prescriptions in Navarro, Frenk, \& White (1997), calculate the shear profile for the cluster, and fit it over a $7^{\prime}$ field-of-view with a SIS profile to mimic the observations. Figure 1 shows that based on this model, the high-redshift X-ray selected clusters are comparable in mass to only the highest tip of the mass distribution of the lower redshift cluster population, while the EDisCS sample is much more comparable in its mass range to the lower redshift samples.

\section{Background galaxy number counts}

Because the optical images used in this analysis were all taken with the same telescope and instrument over a relatively short period of time using the same observing strategy, 


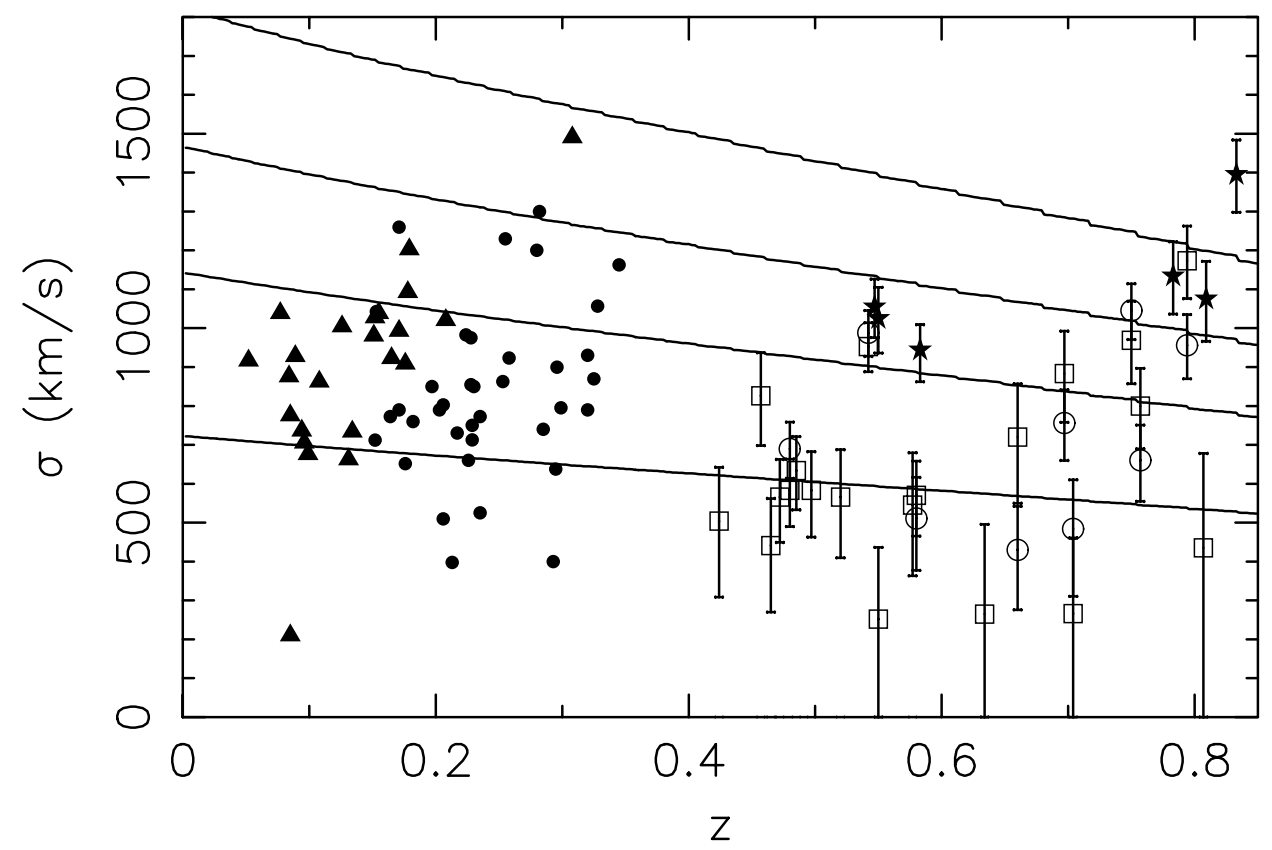

Figure 1. Plotted above are the best-fit SIS velocity dispersions for the EDisCS clusters from VLT images (open squares) and ACS images (open circles), high- $z$ X-ray selected clusters from Clowe et al. (2000) (stars), and lower redshift X-ray selected samples from Dahle et al. (2002) (filled circles) and Cypriano et al. (2004) (filled triangles). The error bars are given as $1 \sigma$, but the measured quantity is proportional to the square of the velocity dispersion. The lines show a model for the evolution of the weak lensing measured velocity dispersion with redshift.

the variation in the number density of faint, background galaxies and their rms shear measurements between the fields should be mostly a function of PSF size, exposure time, bandpass, and cosmic variance without any systematic errors from varying telescope and camera optics. As such, this data set provides an excellent database to study the effects that the various observing conditions can have on expected noise levels in future ground-based surveys.

In Table 1 we give the number density of background galaxies rms shear variance in the final galaxy catalog used for the cluster mass reconstructions. In Figure 2 we show the number density of background galaxies and a shear-field noise estimate (as rms shear per sq. arcmin per shear component) as a function of PSF FWHM for each observed passband and the combined catalog. In order to calculate the rms shear variation for the galaxies, we first subtracted a smoothed shear-field, using a 10" Gaussian smoothing kernel, from each galaxies shear measurement as a first-order correction to remove the cluster shear signal which would otherwise cause the fields around higher-mass clusters to be detected as having a higher noise level.

As can be seen, in general the deeper 2-hour exposures do provide a small increase in the number density of background galaxies over the 45-minute exposures with similar PSF FWHM. Reducing the size of the PSF, however, results in a much larger increase in the number density of usable background galaxies, with 0.' 6 FWHM, 45-minute exposure time images having the same or more usable background galaxies than 0.7 FWHM, 2hour exposure time images. The exception is in the $I$-band, where the longer exposure images have similar numbers of background galaxies as the shorter. This appears to be due to three factors: First, the initial galaxy catalogs were created from a combined 


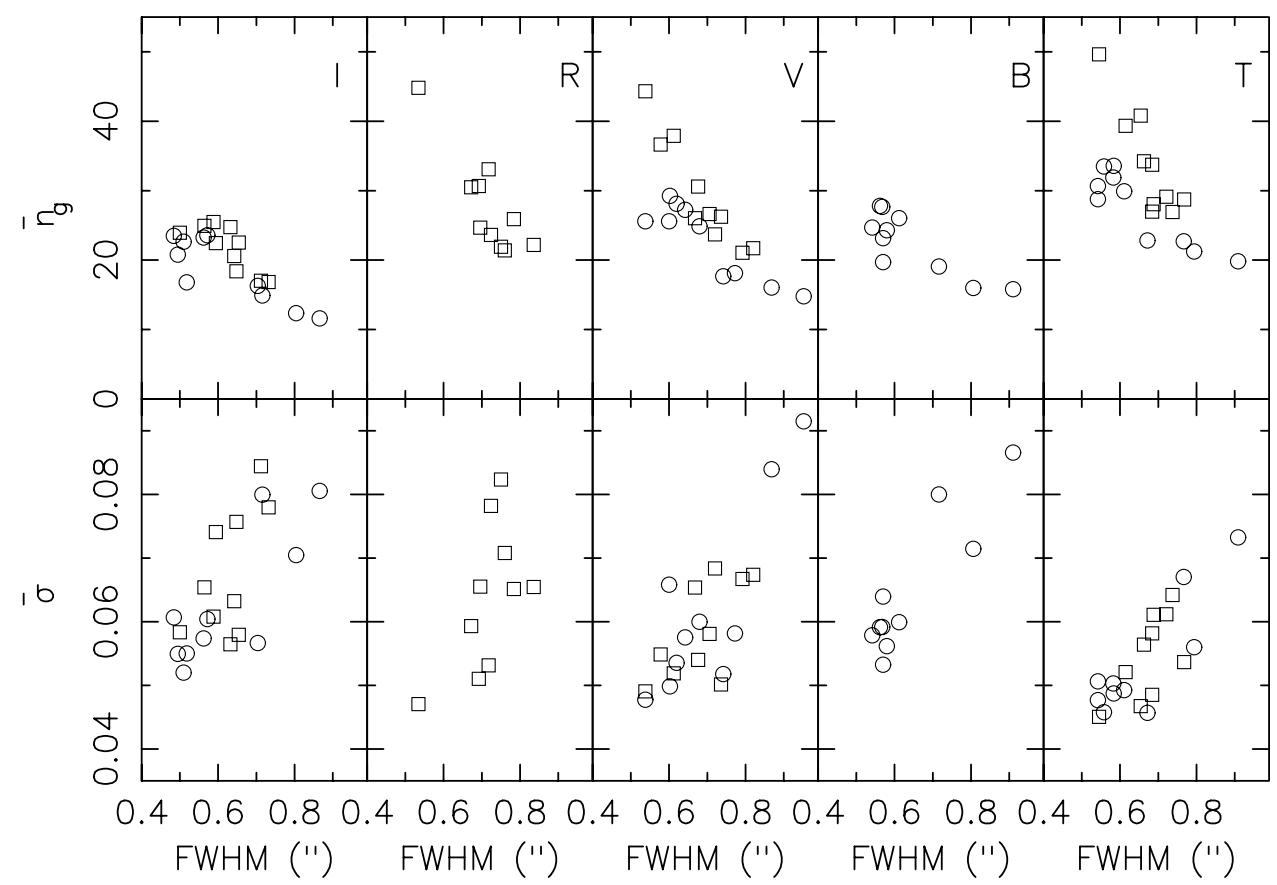

Figure 2. Plotted above are the number density of background galaxies usable for shear measurements (top panels) and the rms shear noise-level per sq. arcminute per shear component from these galaxies (bottom panels) as a function of PSF size for the four observed passbands $(I, R, V$, and $B)$ and the combined catalog $(T)$ from the VLT images. The 2-hour exposure time images of the high- $z$ clusters are plotted as squares, while the 45-minute exposure time images of the lower redshift clusters are plotted as circles.

image in which all of the passbands were coadded using the inverse of the square of the sky noise level as a weighting factor. As a result, the $I$-band images contributed less weight to the final image than did the other passbands, and therefore while the catalogs contain the highest number density of galaxies from any other image combination, the catalogs contain fewer faint red objects than would be included in a strict $I$-band detected catalog. The additional galaxies in the $I$-band detected catalog, however, are likely to be at redshifts similar to the clusters, and therefore would not contribute much to the weak lensing signal. The second effect is that many of the galaxies which are picked up in the longer exposure time catalogs have sizes which are larger than stars in the bluer passbands but are consistent with stars in $I$, and therefore did not have their shapes measured in $I$. The smaller size in $I$ than the other passbands is likely due either to the Mexican-hat filter method for determining $r_{g}$ preferring smaller values at higher sky-noise levels for a given object, or possibly to the background galaxies possessing blue outer regions. The final effect is that the 2-hour exposures were taken in fields with higher redshift clusters on average than the 45-minute exposures, and as a result the color cuts to remove the cluster galaxies in the 2-hour exposures would also have removed $z \sim 0.7-0.9$ field elliptical galaxies which are still present in the 45-minute exposure catalogs.

When looking at the rms shear noise-levels, however, the results seem to be a function only of PSF FWHM and independent of the exposure time. This is a result of the extra galaxies detected in the longer exposure time images having a greater variance in their shear measurements, which offsets the increased number density of the galaxies. This is likely to be due to three factors: First, the fainter objects are, on average, smaller 
and therefore have increased measurement noise from the PSF correction. Second, the fainter objects have a higher mean redshift and are thus observed in a bluer restframe passband, so are more likely to have their luminosity dominated by starbursts and thus have a higher intrinsic ellipticity. Finally, because the fainter objects are at higher mean redshift, they are affected more by the cluster's gravitational shear, but are corrected at the same level as the lower redshift galaxies. As a result, the higher redshift background galaxies will still have a small increase in their rms shear variance as they are still being affected by the lensing induced shear. This last effect, however, should be very minor for all but the most massive of the clusters.

It should be noted that the noise estimate in Figure 2 is valid only for a shear which is applied independent of the redshift of the background galaxy. For high redshift clusters, which cause a significantly greater shear in $z \sim 3$ galaxies than $z \sim 1$ galaxies, including the additional high-variance galaxies detected in the deeper exposures does increase the signal-to-noise of the lensing measurement.

There are a number of large-scale, ground-based optical surveys which are either currently underway, such as the CFHT Legacy Survey (Mellier 2004), or in planning stages, such as the LSST (Tyson 2002) and Pan-STARRS (Kaiser et al. 2002). Almost all of these surveys have plans to measure the weak lensing shear in the images for use in, eg, measuring the power-spectrum of mass in the nearby universe and detecting and characterizing structures by mass. The results given above suggest that shear signal in these surveys will be best measured by combining the individual images of a given field which have the lowest possible FWHM for the PSF, even if it means not using the majority of the raw data in the image co-addition process (and presumably combining high-seeing images together to obtain additional measurements of the shear from the larger background galaxies). This also implies that the surveys which plan to build deep images by taking many shallow exposures separated by large amounts of time will be better served by a site which delivers excellent quality seeing (FWHM $<0$ ". 6 ) for a small, but not negligible, fraction of the time, even if it has a significant tail in the seeing distribution toward much larger PSF size than they will by a site which delivers consistent, mediocre ( FWHM $\left.0^{\prime \prime} 8\right)$ image quality.

\section{References}

Bertin, E. \& Arnouts, S. 1996, A\&AS 117, 393.

Clowe, D., Luppino, G. A., Kaiser, N., \& Gioia, I. M. 2000, ApJ 539, 540.

Cypriano, E. S., Sodré Jr., L., Kneib, J.-P., \& Campusano, L. E. 2004, ApJ submitted.

Dahle, H., Kaiser, N., Irgens, R. J. et al. 2002, ApJS 139, 313.

Fontana, A., D’Odorico, S., Fosbury, R. et al. 1999, A\&A 343, L19.

Gonzalez, A. H., Zaritsky, D., Dalcanton, J. J., \& Nelson, A. 2001, ApJS 137, 117.

Kaiser, N., Aussel, H., Burke, B., et al. 2002, in: Tyson, Wolff, \& Sindey (eds.), Survey and Other Telescope Technologies and Discoveries. Proceedings of the SPIE, Volume 4836, pp. 154.

Kaiser, N., Squires, G., \& Broadhurst, T. 1995, ApJ 449, 460.

Mellier, Y. 2004, in: F. Combes et al. (eds.), SF2A-2004: Semaine de l'Astrophysique Francaise. EdP-Sciences, Conference Series, pp. 8.

Mo, H. J. \& white, S. D. M. 2002, MNRAS 336, 112.

Navarro, J. F., Frenk, C. S., \& White, S. D. M. 1997, ApJ 490, 493.

Tyson, T. 2002, in: Tyson, Wolff, \& Sindey (eds.), Survey and Other Telescope Technologies and Discoveries. Proceedings of the SPIE, Volume 4836, pp. 10.

Wechsler, R. H., Bullock, J. S., Primack, J. R. et al. 2002, ApJ 568, 52. 